\title{
Erratum to: Deriving respiration from photoplethysmographic pulse width
}

\author{
Jesús Lázaro • Eduardo Gil · Raquel Bailón · \\ Ana Mincholé • Pablo Laguna
}

Published online: 15 December 2012

(C) International Federation for Medical and Biological Engineering 2012

\section{Erratum to: Med Biol Eng Comput \\ DOI 10.1007/s11517-012-0954-0}

Due to an equations formatting error, the presentation of some expressions was incorrect. A list of these expressions is given below:

Sect. 2.3, ninth paragraph: $d_{\mathrm{s}}^{\mathrm{P}}(n)$ should be read as $d_{\mathrm{BRV}}^{u}(n)$.

Sect. 3.1, second paragraph: $d_{\mathrm{s}}^{\mathrm{P}}(n)$ should be read as $d_{\mathrm{PWV}}^{u}(n)$.

Table 2: The correct table is given at the end of this erratum.

Sect. 2.4, third paragraph: $f_{\mathrm{s}}^{\mathrm{P}}(j, k)$ should be read as $f_{\mathrm{P}}^{\mathrm{I}}(j, k)$.

Sect. 2.4, fifth and sixth paragraphs: The corrected paragraphs are given below.

In the averaged spectrum $\bar{S}_{k}(f)$ the algorithm also searches the largest peak [denoted $f_{p}^{\mathrm{I}_{a}}(k)$ ] and $f_{p}^{\mathrm{II}_{a}}(k)$ defined as the nearest to $f_{\mathrm{R}}(k-1)$ inside the interval $\Omega_{\mathrm{R}}(k)$ which is at least larger than $85 \%$ of $f_{p}^{\mathrm{I}_{a}}(k)$. At this time the reference frequency $f_{\mathrm{R}}(k)$ can be updated as:
$f_{\mathrm{R}}(k)=\beta f_{\mathrm{R}}(k-1)+(1-\beta) f_{p}(k)$

where $\beta$ denotes the forgetting factor and $f_{p}(k)$ is defined by

$f_{p}(k)= \begin{cases}f_{p}^{\mathrm{II}_{a}}(k), & \exists f_{p}^{\mathrm{II}_{a}}(k) \\ f_{p}^{\mathrm{I}_{a}}(k), & \text { otherwise }\end{cases}$

Finally, estimated respiration rate $\hat{f}(k)$ is defined as:

$\hat{f}(k)=\alpha \hat{f}(k-1)+(1-\alpha) f_{p}(k)$

$\alpha= \begin{cases}\alpha_{2}, & \exists f_{p}^{\mathrm{II}_{a}}(k) \\ \alpha_{1}, & \text { otherwise }\end{cases}$

where $\alpha_{2} \leq \alpha_{1}$, providing more memory when $f_{p}^{\mathrm{II}_{a}}(k)$ could not be set.

Table 2 Percentage of utilization of each DR signal in combination of PRV, PAV and PWV

\begin{tabular}{llll}
\hline Group & \multicolumn{4}{l}{ Percentage of use (\%) } \\
\cline { 2 - 4 } & PRV & PAV & PWV \\
\hline $\bar{f}_{\text {RES }} \geq 0.15 \mathrm{~Hz}$ & 48.24 & 37.80 & 67.63 \\
$\bar{f}_{\text {RES }}<0.15 \mathrm{~Hz}$ & 59.77 & 61.27 & 42.41 \\
All & 52.31 & 46.08 & 58.73 \\
\hline
\end{tabular}

The online version of the original article can be found under doi:10.1007/s11517-012-0954-0.

J. Lázaro $(\varangle)$ · E. Gil · R. Bailón · A. Mincholé · P. Laguna Communications Technology Group (GTC), Aragón Institute of Engineering Research (I3A), IIS Aragón, Universidad de Zaragoza, Zaragoza, Spain e-mail: jlazarop@unizar.es

J. Lázaro · E. Gil · R. Bailón · A. Mincholé · P. Laguna Centro de Investigación Biomédica en Red en Bioingeniería, Biomateriales y Nanomedicina (CIBER-BBN), Zaragoza, Spain 